\title{
What's the point in restaurant calorie counts?
}

\author{
Edward Davies US news and features editor, BMJ
}

New York

There are a lot of fat people in America. More than a third of US adults are obese according to data from the Centers for Disease Control and Prevention. In 2008, medical costs associated with obesity were estimated at \$147bn (£97bn; $113 \mathrm{bn}$ ); the medical costs for people who are obese were $\$ 1429$ higher than those of normal weight. Between 1988-94 and 2007-8 the prevalence of obesity increased in adults at all income and education levels. It's a big problem, no pun intended.

So it's no surprise that measures are progressing to reverse this problem, and one of the more recent campaigns has been a push to require chain restaurants to list calorie counts on their menus. In the Patient Protection and Affordable Care Act, the US Food and Drug Administration (FDA) began the process of requiring calorie labeling for "restaurants and similar retail food establishments that are part of a chain with 20 or more locations doing business under the same name and offering for sale substantially the same menu items." The guidelines have been a source of controversy ever since.

The evidence over the efficacy of such moves is mixed and an understandable reluctance from various quarters of the food industry probably doesn't greatly help the cause.

This week the $B M J$ publishes research that will probably only add to the uneven picture rather than solve the conundrum.
Investigators researching estimations of calorie content in consumers' meals from fast food restaurants found a routine and sizable underestimation-so far so positive for calorie labeling (doi:10.1136/bmj.f2907). But despite the conclusion that labeling "might" reduce the underestimation it was hard for them to be more concrete as they also found that "noticing calorie information in the restaurant had no effect on the accuracy of calorie estimations."

There's no doubt that on an anecdotal level some people do find calorie labeling helpful, but if we're tackling a public health problem and the population level data show questionable benefit, is this part of the Affordable Care Act worth the continuing controversy?

The implementation of the Affordable Care Act is one of the big ongoing topics of 2013 and this week we also publish an international view on it from four visiting academics, currently researching in the US (doi:10.1136/bmj.f3261). Their conclusions on the challenges facing Medicaid expansion hardly make for a walk in the park either, but then, if health reform were straightforward, it would have been done already.

Cite this as: BMJ 2013;346:f3540

๑ BMJ Publishing Group Ltd 2013 